\title{
46,XY Disorder of Androgen Action
}

National Cancer Institute

\section{Source}

National Cancer Institute. 46,XY Disorder of Androgen Action. NCI Thesaurus. Code C120194.

Conditions in 46,XY individuals in which androgens are produced in typical amounts, but tissue response to androgens is reduced. 